\title{
Research On Structural Dynamics Of Safe Operation Of The Large Wind Turbine
}

\author{
Zhiqiang Xu \\ Department of Physics and Technology, Kunming University, Kunming 650214, China \\ 2793436659@qq.com
}

Keywords: Wind Turbine; Dynamics; Load; Analysis

\begin{abstract}
The modal analysis is conducted to the base -tower. The effects of stiffness changes in different directions of the pile foundation on the natural frequency of the wind turbine is studied. After the analysis to the harmonic response of the base-tower, the response of the structure of the tower at different locations at different excitation frequencies. It is shown from the analysis of dynamics of the wind turbine that different structures in response to the same dynamic loading are different because the size of the response has something to do with the natural frequency of the structure.
\end{abstract}

\section{Introduction}

With the development of the industrial revolution, the electric motors, generators, power transmission equipment have gradually been invented. Aerodynamics is now mature with the enthusiasm of the sky exploration. These scientific progress has laid the foundation for the development of the wind turbine. However, with utilization of coal, oil, natural gas, the research on the wind power was at a low point. Only after the global oil crisis was the development and utilization of wind power started, following the industrialized and large-scale way. There are various types of the wind turbine. They can be generally divided into horizontal axis wind turbines and vertical axis ones in accordance with different wind wheel spindles and the wind directions; according to different action principles, they can be divided into lift-type wind turbines and drag-type ones. The spindle of the wind wheel and the wind turbine parallel to the wind direction is called horizontal axis wind turbine unit, including the types of propeller, wing sail and multi-airfoil. The wind wheel should be parallel to the wind direction in the horizontal axis wind turbine unit. Vertical axis wind turbine unit means the spindle of the wind wheel is at the right angle with the wind direction. Since the vertical axis wind turbine unit can rotate for the wind in any direction, there is no need for luffing devices.

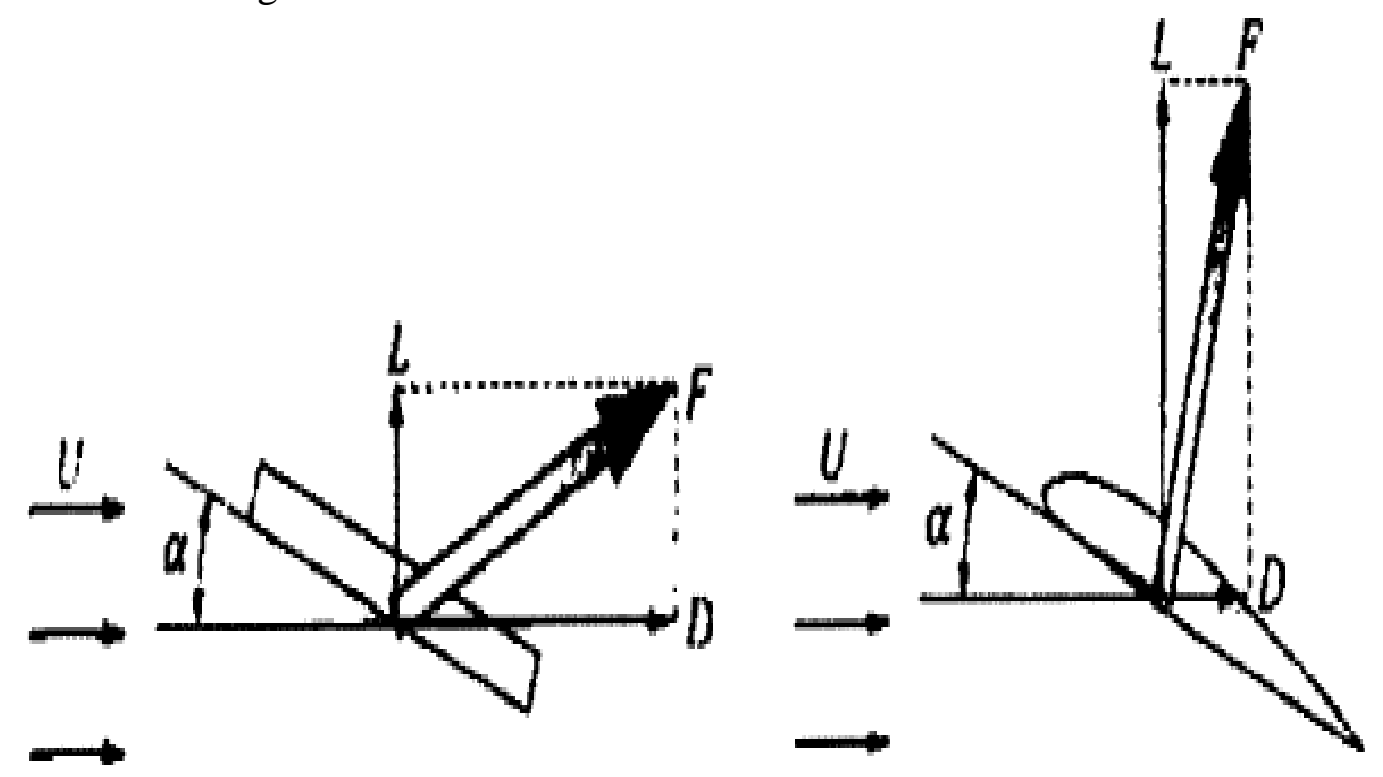

Fig1. Lift and drag 


\section{The Performance Coefficient Of The Wind Turbine}

The manifestation of the wind energy is the kinetic energy. It can be expressed as the product of a quadratic of the air quality and the of the speed

$$
E_{W}=\frac{1}{2} m V^{2}
$$

In the formula, $\mathrm{V}$ is the wind speed, $\mathrm{m}$ is that the air quality of the area of the wind through the wind wheel within the unit of the time, that is:

$$
m=\rho A V
$$

In the formula, $\rho$ is the density of air. From the above two equations, the wind energy of the sweeping area through the wind turbine blades within the unit of the time can be obtained:

$$
E_{W}=\frac{1}{2} \rho V^{2}(A V)=\frac{1}{2} \rho A V^{3}
$$

The sweeping area of the blades rotation in the horizontal axis wind turbine is the windward area. The atmosphere quality refers to the amount of the windward area on the surface of rotation of the wind wheel From the analysis of the above equations, the wind energy is proportional to the cube of the speed. So the speed of the wind in the wind turbine rotor sweeping area is one of the important factors for the wind turbine to obtain wind energy. If the energy obtained from the wind in the unit of time for the wind turbine is called $\mathrm{P}$, and the ratio between the power of the wind turbine unit $\mathrm{P}$ and wind power is called the power coefficient, which is expressed as $C_{\mathrm{p}}$

$$
C_{P}=\frac{P}{E_{\text {W }}}
$$

\section{The Analysis Of Safe Operation Parameter}

During the operation, the force of the wind turbine is very complex. The tower interacts with the dynamic load which the blades rotate generate. The vibration displacement generated by the load can affect the normal operation of the wind turbine unit. Besides, It may lead to the fatigue damage of the tower, instability and even collapse [6] [7]. To ensure the safe operation of the wind turbine , static and dynamic analysis must be carried on to the tower of the wind turbine. Therefore, the research on the aerodynamics related to the tower performance of the large-scale wind turbine and structural dynamics has aroused wide concern[1] [2]. In dynamic analysis, selecting the boundary conditions has a greater impact on the results [9]. Document[3] and [4] studied the carrying capacity of the deep drum wind turbine foundation. Document [5] uses Cone pendulum model to simulate the ideal soil-base structure. Document[8] analyzes the response characteristics of the wind turbine under seismic loading, etc. On this basis, this article has established the coupling model foundation among the base, the tower and the top quality. It has also analyzed the dynamic response of the base-tower under a variety of boundary conditions, as well as the influence that the pile has on the tower modes within a certain range of the rigidity.

To ensure the safe and reliable operation of the wind turbine structure, it's necessary to analyze dynamic of the wind turbine. Different structures responding in the same dynamic loading are different because size of the response is connected with the inherent frequency of the structure [10]. Transient dynamic analysis is adopted to determine structural dynamics of load which changes with time. The modal superposition method is used to analyze transient response when the finite element software ANSYS is applied. The core is to utilize the orthogonal relation. Then turn the kinematic equation into $\mathrm{n}$ mutually independent equations. Thus, calculate the contribution of each the mode. This method is suitable for solving a small number of low order modes play a major role or the desired dynamics analysis calculating the response time is longer in case of problems. Wind load is the major wind turbine structure is subjected to load, which can be divided into the average wind and the fluctuating wind. The cycle of the average wind is generally much larger than that of the natural vibration of the structure, which acts as a static load; the fluctuating wind is a Gaussian 
random process with zero mean, whose changing cycle is often close to that of the structural vibration. It should be treated as a dynamic load in the analysis. The structural dynamic response of the wind turbine is analysis, simulating the average speed of the wind at $12 \mathrm{~m} / \mathrm{s}$ at the hub during 0-40 seconds and calculating the dynamic response of the wind turbine. When the blades are in different positions, the displacement amplitudes at the tip of each blades vary greatly. The tip amplitude of the top blade is $1.7 \mathrm{~m}$, the amplitude of the lower blades is close to $0.8 \mathrm{~m}$. The middle displacement at the hub and the tower is smaller wheels at the tower, so are the amplitude variations. In engineering practice, because the cabin elevation and blades use the pre-curved technology, touching the tip of the tower can effectively be avoided. The blade is a flexible body and the stiffness of other parts are larger. Under the dynamic response of the wind turbine, the speed at the tip of the blade and acceleration are larger than that in other locations and the amplitude is the most violent. Since the rotating of three blades and the change of the azimuth make different vibration responses in different locations, the connection stiffness between the wind wheel and the tower has a greater impact on the hub as well as the tower. Thus, when the external excitation reaches the natural frequency of the blade, the blade vibration will continue to increase. The blade flutters, which will cause serious damage to the blade. Therefore, the analysis of the dynamic response of the complete machine will improve the reliability of the wind turbine dynamics.

\section{Conclusion}

The modal analysis has been conducted to the wind turbine system by using ANSYS finite element software. The effect of the blade of the wind turbine at different azimuth has on the natural frequency has been studied. The stability analysis shows that resonance does not occur among the major components of the wind turbine. Campbell diagram analysis of the wind turbine system suggests that the tower belongs to the soft tower and low-order frequency of the system does not coincide with the rotational frequency and the blade passing frequency. The system is stable. However, during the start-up of the wind turbine, the blade frequency coincides with the low-level frequency of the wind turbine at the rotational speed of 6 to 8rpm . Therefore, in the start-up process of the wind turbine, the above regions should be quickly skipped. Through transient response analysis, different location under the wind load of the wind turbine, displacement, velocity and acceleration change with the time. The results show that since the blades are two flexible bodies, while the rigidity of other parts is greater, the displacement at the tip, velocity and acceleration are larger than at other locations. In addition, the vibration is the most severe. The deformation at the blade tip is caused by the wind load at the three blades and the elastic deformation coupling of the tower

\section{References}

[1]G.G.Gebhardt, S.Preidikman, J.C.Massa. Numerical simulations of the aerodynamic behavior of large horizontal·axis wind turbines[J].Hydrogen Energy, 2010,35(1):6005-601 1.

[2]Luong Van Binh,Takeshi Ishihara,Pham Van Phuc,et a1.A peak factor for non-Gaussian response analysis of wind turbine tower[J].Journal of Wind Engineering and Industrial Aerodynamics,2008,96:22 1 7-2227.

[3]Puyang Zhang,Hongyan Ding,Conghuan Le,et a1.Test on the Dynamic Response of the Offshore Wind Turbine Structure with the Large-Scale Bucket Foundation[J].Procedia Environmental Sciences,2012,12,Part B:856-863.

[4]S.Adhikari,S.Bhattacharya.Dynamic analysis of wind turbine towers on flexible Foundations[J]. Shock and Vibration, 2012,19(1):37-56.

[5]M.Harte,B.Basu,S.R.K.Nielsen. Dynamic analysis of wind turbines including soil-structure interaction[J]. Engineering Structures,2012,45:509—518. 
[6] Jianlong Si, Gang Tu, Fengya Shen, etc. Calculating the horizontal displacement of the tower top of large-scale wind turbine [J].Energy Engineering, 2009,134 (2): 28-30

[7] Xi Song, Jianxin Dai. Mechanical modeling of horizontal axis of the wind turbine tower and ANSYS simulation analysis [J]. Gansu Sciences Sinica, 2011,23 (1): 91.95

[8] Zhenyu Wang, Biao Zhang, Zihua Zhang, etc. Study on dynamic characteristics of the tower base of 1.5MW wind turbine [J]. Energy engineering, 2011,2: 29.36

[9] Jiaojiao Wang, Gang Shi, Yongjiu Shi, etc, Analysis on considering the natural frequency of the wind turbine tower under different boundary conditions of points [J] Special structure, 201l, 28 (5): 5-8

[10] Hole Yi Gang, Gu Hao. Load analysis and power control based on wind shear and tower shadow effect of the large-scale wind turbine [J]. East Southern University Journal, 2010,40 (1): 229.23 